\title{
GOLD NANOPARTICLES: A PROMISING THERAPEUTIC APPROACH
}

\author{
Harsharan Pal Singh ${ }^{1}$, Ashmeet Kaur ${ }^{2}$, Ishpreet Kaur ${ }^{2}$, Harpal Singh Buttar ${ }^{3}$, and \\ Sukhwinder Kaur Bhullar ${ }^{4,5}$ \\ ${ }^{1}$ Department of Quality Assurance, AIMIL Pharmaceuticals (I) Limited, New Delhi, India \\ ${ }^{2}$ Department of Quality Assurance, Delhi Institute of Pharmaceutical Sciences \& Research, \\ New Delhi, India \\ ${ }^{3}$ Adjunct Professor, Department of Pathology \& Laboratory Medicine, Faculty of Medicine, \\ University of Ottawa, Ontario, Canada \\ ${ }^{4}$ Department of Mechanical Engineering, Bursa Technical University, Bursa, Turkey \\ ${ }^{5}$ Department of Mechanical Engineering, University of Victoria, Victoria, BC, Canada
}

\begin{abstract}
Nanotechnology is rapidly advancing and will leave no field untouched by its ground breaking innovations. Nanoparticles are molecules with a diameter ranging from 10-100 nm. Nanotechnology has promising biomedical applications and most noteworthy amongst them are noble metal particles. For instance, gold nanoparticles (AuNPs) provide a unique blend of physical and optical properties, chemical inertness, and high surface to volume ratio. They can be synthesized as well as functionalised to support various ligands on their surface. Their surface functionalization and diverse properties render the gold nanoparticles highly useful for drug delivery and gene carrier for therapeutic purposes and as molecular probes for disease diagnosis. The foundation for the usage of AuNPs in therapeutics and diagnosis was laid by the ancient studies done with ruby gold for curing diseases in middle ages. Presently, AuNPs have become available in different types such as spheres, rods, shells, cages and SERS particles which vary in shape, size and physical properties. The biomedical applications of these particles include drug and gene delivery, cancer diagnosis and therapy, determination of biological molecules and microorganisms, detection of disease etiology, immunoassay, enzyme immobilization, etc. Overall, the focus of this review is to highlight that AuNPs provide an excellent platform for the discovery of new therapies, cure for certain cancers, molecular probe for diagnostic purposes, as well as gene carriers and drug delivery vehicles. Biomed Rev 2015; 26: 23-36.
\end{abstract}

Key words: gold nanoparticles, cancer treatment, drug delivery system, gold nanocarrier therapy

Received 2 December 2015, revised 12 December 2015, accepted 14 December 2015.

Correspondence to Mr. Harsharan Pal Singh, Department of Quality Assurance, AIMIL Pharmaceuticals (I) Limited, New Delhi, India Tel.: +91-9999054899, E-mail: harsharanpal.singh@gmail.com 


\section{INTRODUCTION}

Nanotechnology is a multifaceted research field that deals with the nanometer sized entities ranging from 10-100 nm (1), and can be defined as the characterization, design, production and application of devices, structures and systems by controlling shape and size at a nanometer scale. It embraces diverse fields like chemistry, physics, material sciences, molecular biology, and medicine. Recently, potential uses of nanomaterials have attracted ample amount of attention of basic and applied scientists $(2,5,7,8)$. Although the nanoparticles size ranges from one to several hundred of nanometers, they are comparatively smaller than large biomolecules such as antibodies, enzymes and receptors as well as neurons and red blood cells $(1,2-8)$. The nano-sized particles have unique optical, magnetic, electronic and structural properties (9). This property makes the nanoparticles highly promising for a wide range of biomedical applications such as molecular diagnosis, cellular imaging and targeted drug delivery depending upon the composite shape or structure of the nanoparticles (10). The well-studied nanoparticles include carbon nanotubes, liposomes, quantum dots, magnetic nanoparticles, polymeric particles and metallic nanoparticles $(7,11)$. Namely, the unique and distinctive characteristics of nanoparticles are: (a) small size which ranges from 1-100 nm, (b) large surface-to-volume ratio, (c) shape, size and composition which determines the physical and chemical properties, (d) qualitative and quantitative target-binding properties, and (e) some nanostructures show high robustness (3).

The applications of metal nanoparticles in medicine and biomedical research are rather a new phenomenon and an overwhelming number of publications have appeared in the world literature during the past 10 years. A few unique properties of nanoparticles are their high reactivity towards the living cells, stability at high temperatures, and translocation into the cell organelles. These particles also demonstrate unique optical properties which make them capable of producing quantum effects suitable for imaging. These characteristics of nanoparticles make them highly useful in diagnostics and therapeutics. Among all the metal nanoparticles, the most commonly studied are gold, silver, iron, and titanium oxide nanoparticles (9).

Gold nanoparticles were used by the Romans for the purpose of decorating and staining of glass. It is nearly 150 years ago when Michael Faraday noticed that the properties of colloidal gold solution were different from that of bulk metalic gold. Over the last half-century, different high yielding techniques for the synthesis of gold nanoparticles (AuNPs, nanogold), including spherical and non-spherical shapes have been developed (12).

The AuNPs are biocompatible and non-toxic which makes them more advantageous over other metal nanoparticles. They have physico-chemical inertness, and are easy to synthesize and fabricate. The AuNPs exhibits optical properties related to plasmon resonance as well as functionalisation with molecular probes. These unique physico-chemical properties make them compatible for broad range of biomedical applications. Presently, the biomedical applications of AuNPs have expanded to divergent fields like genomics, microorganism detection and control, targeted drug delivery, optical imaging, biosensors, immunoassays, monitoring of biological activity of cells and tissues, exploiting resonance scattering, photo-thermolysis of cancer cells, and in vivo photo-acoustic techniques $(9,12,13)$.

\section{HISTORICAL PERSPECTIVE OF GOLD NANOPARTICLES}

Metallic gold has a melting point of $1064^{\circ} \mathrm{C}$ and a boiling point of $280^{\circ} \mathrm{C}$. This precious metal does not react with water or oxygen and is an excellent conductor of electricity (14). In 1857, Michael Faraday explored the ruby gold nanoparticles and was astonished by the ruby color of the colloidal particles. His studies on the Au particles led to the birth of the modern day colloidal chemistry and the foundation of nanotechnology. Faraday observed that metallic Au was dispersed uniformly in both ruby glass and ruby fluid which was based upon its physico-chemical characteristics. Some 100 years later these ruby-colored colloids were found to be stable and their size was determined with the aid of electron microscope to be in ranges of 2-6 $\mathrm{nm}(15,16)$.

Zsigmondy who started to investigate upon the opacity and color of the ruby glass, tried to reproduce Au colloids through different methods. He combined Faraday's work and introduced a new procedure called, 'seed mediated method'. This method is still being used for the synthesis of nanoparticles. He also came up with an ultra-microscope for characterization of size, shape and structure of nanoparticles. Another scientist, Svedberg introduced ultracentrifuge and through it demonstrated the dependence of the motion of macromolecules (colloids) on their shape and size (15).

In the middle ages, colloidal gold was used for curing wide variety of diseases such as arthritis, heart disorders, epilepsy, dysentery, venereal diseases and tumors and also for the diagnosis of syphilis, a method which was used until the 20th century. Towards the end of 16 th century, colloidal gold was 
routinely used to make ruby glass and for coloring ceramics by techniques which are still used for such purpose (14).

\section{SYNTHESIS OF GOLD COLLOIDS}

Gold nanospheres were the first ones to be discovered and studied, followed by the rest of the subtypes. Based upon shape, size and physical properties the AuNPs are categorised into different subtypes: nanospheres, nanorods, nanoshells and nanocages Also, AuNPs which have great surface-enhanced Raman-scattering properties are called "SERS nanoparticles" $(1,13,15)$. (Fig. 1)

\section{GOLD NANOSPHERES}

It was proposed a method for the synthesis of gold nanospheres of $2 \mathrm{~nm}$ to $100 \mathrm{~nm}$ diameter which was based on the reduction of aqueous tetrachloroauric acid $\left(\mathrm{HAuCl}_{4}\right)$ with sodium citrate in water at $90-100^{\circ} \mathrm{C}$. The reducing agent and the experimental condition can be varied as desired. As this procedure is reasonably simple and environmentally friendly, it is the most commonly used method for the synthesis of nanospheres. By varying the molar citrate:gold ratio, the size of the nanospheres can be restrained $(1,14,16,17)$. Usually, small amounts of citrate will generate larger nanospheres $(1,14)$. The nanospheres obtained through this process generally have a broad distribution of size and shape. Mostly, the nanoparticles produced fall in the size range of $12-60 \mathrm{~nm}$ with a relative size distribution of $10-15 \%$ and show a non-uniform and irregular shape such as ellipsoids, quasi-spheres, and triangles (16). However, this method has limitation of restricted use of water as the solvent and low yield $(1,14)$.

A second method known as 'Burst method' is capable of producing thermally and air stable nanospheres which are more mono-dispersed and their size can be controlled up to $<10 \mathrm{~nm}$ of diameter $(1,14,17)$. In this method, $\mathrm{AuCl}_{4}^{-}$is transferred using a phase transfer reagent tetraoctylammonium bromide from the aqueous solution to toluene and then reduced in the presence of dodecanethiol $\left(\mathrm{C}_{12} \mathrm{H}_{25} \mathrm{SH}\right)$ with aqueous sodium borohydride. The ratio of thiol: gold controls the size outcome of the nanospheres (16). A larger thiol:gold ratio and the faster addition of the reducing agent under cold conditions will produce smaller and more mono-dispersed nanospheres $(1,14)$.

\section{GOLD NANORODS}

There is a wide variety of strategies proposed for the synthesis of Au-nanorods. The most commonly used method for production of Au-nanorods is the 'Seed-mediated synthesis'. The chemical reduction of gold salt takes place in the presence of a strong reducing agent like sodium borohydride $\left(\mathrm{NaBH}_{4}\right)$ forming gold seeds. Then, these seeds are added to a gold salt solution containing a weak reducing agent such as hexadecyltrimethylammonium bromide and ascorbic acid for continuation of growth. The Au seeds formed in the solution with strong reducing agent acts as site for nucleation for formation of nanorods $(1,14,17-19)$. The ratio of gold seeds:gold precursor is important for controlling the length to width ratio of the nanorods $(20,21)$. Furthermore, with the addition of $\mathrm{AgNO}_{3}$ to the solution, the yield could be greatly increased (22). The electrochemical deposition of gold into pores of the nanoporous polycarbamate or alumina template membranes is an emblematic method of synthesis of nanorods. This method is called 'template method'. The pore diameter of the template membrane is the pre-determinant for the nanorod diameter, while the amount of gold deposited within the membrane pores is the pre-determinant for the nanorod length. A low yield due to formation of only a single monolayer of the $\mathrm{Au}$-nanorods is a fundamental disadvantage of this method.

Apart from the above described methods, several other procedures: namely growth of nanorods on mica surface,

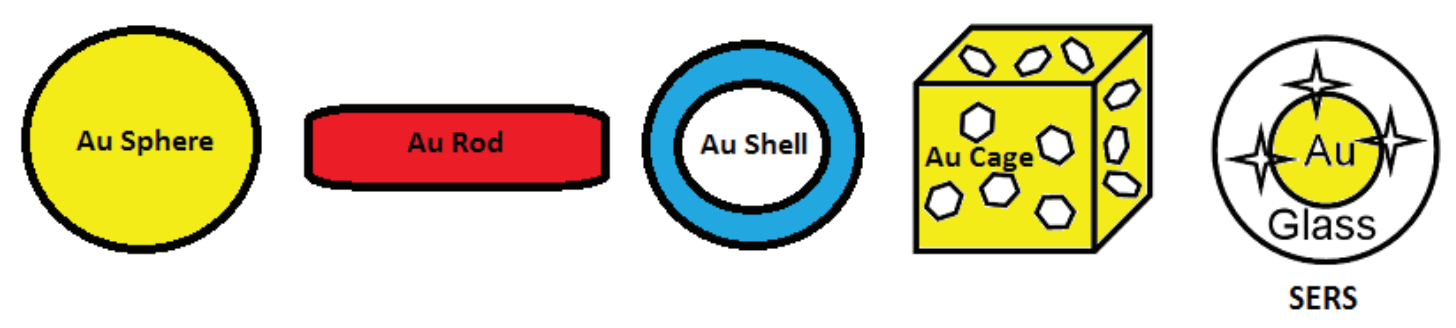

Figure 1. Schematic illustrations of different types of gold nanoparticles. 
bio-reduction, and photochemical synthesis as well as electrochemical synthesis for the growth and fabrication of nanorods have been explored $(1,14)$.

\section{GOLD NANOSHELLS}

Gold nanoshells are a type of spherical nanoparticle which consists of a dielectric core of materials such as silica, polystyrene or sodium sulphide coated by a thin layer of metal (usually $\mathrm{Au})(8,23)$. Silica cores are grown by the reduction of tetraethyl orthosilicate in ethanol through the process developed by Stöber. The silica nanoparticles are coated with gold by using a seeded growth technique. An amine-terminated silane is used as a liner molecule for the attachment of small gold nanospheres of diameter 2-4 nm to the silica core. This allows the additional gold molecules to be reduced until the seed particles are consolidated into a complete shell. The diameter of the silica core and the amount if gold deposited on the surface of the core determines the diameter of the gold nanoshell and the shell thickness, respectively. Other methods of production of gold nanoshell are being continuously investigated. The thermo-sensitive core-shell particles can be used as the template for in situ synthesis. For controlling the thickness of the Au nanoshell along with significant reduction of particle aggregation, the micro-gel can be used as core material $(1,14,19,23)$.

\section{GOLD NANOCAGES}

Gold nanocages containing controlled pore size on their surface were synthesized in 2006 by the galavanic replacement reaction between aqueous $\mathrm{HAuCl}_{4}$ and truncated- silver nanocubes. The silver nanostructures can be developed by polyol reduction, in which $\mathrm{AgNO}_{3}$ is reduced by ethylene glycol to develop silver atoms and then nanocrystals or seeds. The morphology of nanocrystals can be controlled by experimental conditions. Through the addition of more silver atoms the desired seeds can be produced. This can be done by controlling the silver seed crystalline structures in the presence of poly-(vinylpyrrolidone). Poly-(vinylpyyrolidone) is a polymer capable of selectively binding to the surface (14, 19, 24).

The galvanic replacement process is used to remodel the silver nanostructures into internal hollow space within $\mathrm{Au}$ nanostructures. Thus, it acts as a sacrificial template for the synthesis of Au nanocages $(1,9)$. The dimension and wall thickness of the resultant Au nanocages can be precisely controlled by adjusting the molar ratio of silver : $\mathrm{HAuCl}_{4}(1)$.
Au nanocages may provide some major advantages such as: (i) their surface plasmon resonance peaks could be adjusted by changing the ratio of $\mathrm{Ag}$ nanocubes : $\mathrm{HAuCl}_{4}$. Thus, covering the entire spectral region from 500 to $1200 \mathrm{~nm}$, (ii) their absorption coefficients could be varied by controlling the number of truncated corners and void sizes, (iii) the $\mathrm{Au}$ nanocages could still show resonance peaks in the near-IR region with remarkably small size of about $50 \mathrm{~nm}$, and (iv) surface modifications of Au nanocages could be achieved and employed in various biomedical applications (14).

\section{SURFACE ENHANCED RAMAN SCATTERING NANOPARTICLES}

Surface enhanced Raman scattering (SERS) is a technique in which the nanoparticles are labeled with groups having activity in the Raman spectral region. In 2002, a new method was reported in which gold nanospheres of diameter $\sim 13 \mathrm{~nm}$ were modified with a group cyanine-3. To monitor the presence of specific target DNA strands, alkythiol-capped oligonucleotide strands were used as probes. Also, in one study conducted in 2008 , gold nanospheres of diameter $\sim 60 \mathrm{~nm}$ were encased with a Raman reporter and then stabilized with a layer of thiolated polyethylene glycol. This technique appears to have more merits than that of other conventional ones, such as chemiluminescence and fluorescence. It provides better sensitivity, robustness, superior performance in blood, high levels of multiplexing and other biological matrices (13-15).

\section{Cellular uptake and fate of gold nanoparticles}

Cellular uptake of nanoparticles is mainly through endocytosis. For nanostructures, it is considered to be via receptor-mediated endocytosis involving an interaction between cell membrane receptors and ligands on the surface of the NPs (30). The cellular uptake and the intracellular fate of the nanoparticles largely depend on size, surface functionality, shape, charge and hydrophobicity $(25,30)$. The optimal sized nanoparticles have smallest internalization time. The particles of diameter 20-50 nm have most efficient uptake while apoptosis is enhanced in $40-50 \mathrm{~nm}$ range. The variation in curvature of the AuNPs is the reason for shape dependent uptake, rod-shaped AuNPs have lower uptake than their spherical counterparts. The positively charged AuNPs have greater uptake efficiency than that for neutral and negatively charged AuNPs (26-29). In depth understanding regarding the uptake and accumulation of nanoparticles in living systems is vital for the successful usage in pharmaceuticals. The cellular uptake is often influenced by the surface charge of the carrier and hydrodynamic radius of 
nanoparticles. The route of administration also determines the biodistribution of nanoparticles. Generally, the nanoparticles have a more prolonged retention in the lymph nodes, in comparison to delivery via intramuscular, subcutaneous or topical routes (31).

\section{BIOMEDICAL APPLICATIONS OF GOLD NANOPARTICLES As a Drug Delivery System}

Drug delivery systems (DDSs) are used to increase the efficacy of a wide variety of pharmaceutical payloads, including small drug molecules or large biomolecules such as proteins and genetic materials. DDSs provide the positive characteristics to the 'free' payload through enhancing solubility, biodistribution, in vivo stability and pharmacokinetic properties. DDSs can be functionalized with ligands to target a specific tissue or cell type, providing a targeted drug delivery system (32-37). Furthermore, they can be loaded with huge amounts of drugs to act as reservoirs, rendering controlled and sustained release of drugs to maintain the levels within the therapeutic window $(32,33)$. The AuNPs have been effectively utilised as a drug delivery system. The transport and release factors play a critical role in the effectiveness of a drug delivery system. The drug can be loaded onto the nanosystem in two ways, either by covalent conjugation or non-covalent conjugation. Covalent conjugation usually requires intracellular processing of a pro-drug, whereas non-covalent conjugation employs active drug itself $(9,25)$. Out of the several approaches used for fabrication of the AuNPs, the most common ones that are employed for targeted delivery applications includes surface modification of AuNPs with cationic polymers or reactive functional groups (e.g. amine, carboxyl and thiol groups). Another approach is drug encapsulation on AuNPs using layerby-layer (LbL) conjugation. These complexes provide better protection of the encapsulated drug from enzymatic metabolic degradation, allowing prolonged half-life $\left(\mathrm{t}_{1 / 2}\right)$ and improved drug efficacy. Through the LbL approach, the amount of drug loaded can be efficiently controlled depending on the number of layers. Further, the delivery to a target cell can be achieved by immobilization of cell-specific targeting molecule onto the AuNP surface (38).

For therapy, the release of drug molecule from nanoparticle is a prerequisite and can be triggered by internal (e.g. $\mathrm{pH}$ or glutathione, GSH) or external (light) stimuli. Internal stimulus controls the release in a biological manner whereas external stimulus has a spatio-temporal control over the release (16, 39). The release in case of covalent conjugation is well controlled, while drug molecules that are non-covalently conjugated suffer non-specific release or other types of interactions. Endogenously triggered release of AuNPs is based on glutathione (GSH) concentration in the plasma or across the cell membrane $(9,16)$. There exists a substantially higher intracellular GSH concentration (1-10 mM) compared to the extracellular thiol concentration (GSH $2 \mu \mathrm{M}$, cysteine $8 \mu \mathrm{M})$ and this higher intercellular concentration is exploited by the GSH-mediated DDS. The pro-drug bound to the AuNPs can be either released via place-exchange reactions or via disulphide exchange. Moreover, surface cysteine of proteins in the bloodstream can participate in thiol-disulfide exchange, resulting in protein carrier conjugates which can alter bioavailability and pharmacokinetic properties $(3,16$, 39-41). The monolayer of the nanoparticles can provide steric shielding against this interaction, hence enabling their use in vivo. Externally controlled release of payload is a corresponding tool for site and time dependent delivery $(40,41)$. The IR radiation triggers local heating of the metal nanoparticles which can result in the controlled release of the therapeutic agents or dyes bound to the particles. When there is adsorption of the molecule onto the surface of the nanoparticle, the temperature increase would often result in desorption of the less stably conjugated molecule. This property of metal nanoparticles can be utilized in development of light regulated photothermal release system. These systems can be of two types: (i) direct desorption of the payload, and (ii) use of a thermally responsible polymer for triggering the release $(16,17)$.

\section{Application of AuNPs for gene delivery}

Gene therapy is envisioned an excellent approach for the treatment of acquired and genetic diseases. Induction of target gene expression and protein synthesis can be achieved by use of DNA or RNA vectors (25). Viral and non-viral systems have been most frequently used for gene delivery. However, the viral and non-viral systems have limitation for causing immune responses and low transfection efficiency, respectively. Gold nanoparticles are considered suitable candidates for gene delivery, since they not only provide a high surface-to-volume ratio but also have a small size that maximises payload to carrier ratio $(38,39)$.

With PEGylated AuNPs, gene expression is enhanced by about 100 -folds as compared to naked DNA $(9,42-47)$. This is so because the PEGylation under the normal cell culture conditions stabilizes the AuNP/DNA complex by providing it 
with a protective layer for a longer time period. This procedure helps to increase transgene expression and reduces cytotoxicity (38). These functionalized nanoparticles are also used for the delivery of amino acids and small interfering RNA (siRNA) $(9$, 42-49). It has been reported that mixed monolayer protected gold clusters (MMPCs) functionalized to amine containing alkyl chain exhibit effective intracellular plasmid DNA delivery and induction of target protein synthesis. The in vivo gene transfection was determined by charge density of MMPCs and alkyl chain length used in fabrication, whereas the protein induction was ascertained by MMPCs to pDNA ratio. The monolayer coverage of MMPCs and MPCs allow regulation of hydrophobicity and charge to maximize transfection efficiency and minimize toxicity $(38,41)$. A considerably superior transfection vector is provided by the amphiphilic particles which demonstrate that the hydrophobicity of nanoparticles improves the efficiency of cellular uptake and/ or the consequent release of the DNA from endosomal vesicles (39). AuNPs functionalized with polyethyleneimine (PEI) and chitosan provides a higher transfection efficiency for gene delivery, so these are used as a delivery vector with reduced toxicity in rabbit cornea $(9,48,49)$.

The siRNA delivery extensively exploits the use of AuNPs as a nanocarrier. Terminal end of the siRNA was modified to contain a functional thiol group for extensive immobilization of siRNA on the AuNPs. Thiol-modified siRNA through Authiol interactions exhibited a strong adsorption on the surface of AuNPs. When this nanocomplex is transported into the cell, free siRNA is released by the reductive cytoplasmic environment and finally it triggers the RNA interference. In addition, the end-functionalized siRNA can also be coupled to the polymer coated AuNPs through chemical linkages. Acidliable or reducible bonds can be used for selective delivery of conjugated siRNAs under acid-pH or reductive environment of the cells (38).

The photo-cleavable linkers are also used for the delivery of adsorbed genetic material. The property of AuNPs heating on absorption of IR radiation is utilized for cleavage of the sensitive linkers. A photoliable nanoparticle was designed to convert the nature of surface from cationic to anionic upon irradiation. The particles were designed to have a photo-cleavable o-nitrobenzyl ester moiety and a quaternary ammonium salt as the end group. On irradiation, the linker cleaves to create an anionic carboxylate group and releases the DNA (39).

\section{Application of AuNPs for chemical drug delivery}

AuNPs are functionalized with various chemical drug entities and biomolecules for specific destruction of cancer and bacterial cells. Several of the anticancer drugs such as methotrexate, adriamycin, paclitaxel and doxorubicin have been studied for delivery through AuNP conjugation $(3,28)$. Tumor necrosis factor-alpha (TNF- $\alpha$ ), has been investigated for anticancer therapy through the usage of nanoparticles. In the nanoparticle delivery system containing a PEG coated AuNP onto which TNF- $\alpha$ is loaded. This approach leads to maximal tumor damage and minimal cytotoxicity. Also, the nanoparticles based delivery coupled with local heating results in enhanced therapeutic efficacy $(1,14)$.

AuNPs having high surface to volume ratio, inherent low toxicity, and sustainable stability provide them with qualities suitable for the design of new drug delivery strategies. The optimization of characteristics such as non-immunogenicity, and enhanced cellular bioavailability are the key issues which need to be kept in mind during the engineering of particle surface. Such novel approach would serve as a useful alternative tool for the traditional delivery systems. Thus, the AuNPs seem to be an emerging framework for drug and gene delivery systems (38).

\section{Applications of nanotechnology for cancer diagnosis and therapy}

Anti-cancer nanotechnology is a versatile area which has potential applications in battling different types of cancers, including molecular diagnosis, molecular imaging, bioinformatics and targeted drug delivery. The growth of anticancer nanotechnology carries the potential for personalized oncology. Thus, it may someday be helpful in such a way that protein and genetic biomarkers could be used for the diagnosis and treatment of cancer on the basis of molecular profiles of each individual patient (1). Gold nanoparticles are being extensively researched for their applications in cancer diagnosis and treatment. In order to optimize the structures of nanoparticle so as to become more efficient diagnostic and therapeutic tools, it is extremely crucial to have understanding of their behavior in the tumor microenvironment. Penetration and accumulation in the hypoxic centers of cancer cells for therapeutics is often difficult and attributed to the large intercapillary distances and variable blood flow typically in solid tumors. Whereas, the outer shell of solid tumor is often the site for angiogenesis and improper lymphatic flow. Angiogenic blood vessels have gaps of nearly $600 \mathrm{~nm}$. The 
vascularized regions are liable to be 'leaky' and resulting in enhanced permeability and retention effects. Owing to their hyperpermeability, tumor cells passively absorb the nanoparticles leading in high concentration (nearly 10-fold more) of the carrier in the tumor tissue. Also, the photothermal properties of nanoparticles help in the active targeting of the molecules and therapeutic agents into the tumor cells (1,9, $31,38,42-57)$.

\section{Cancer diagnosis}

The optical properties of AuNPs along with their ability to form conjugates have been utilized widely in the diagnosis of cancer. Functionalised AuNPs with oligonucleotides, peptides, flourophores, aptamers, antibodies, organic dyes or other biomolecules have been studied for holding a promising role in imaging of cancer cells (42-49). Oligonucleotide conjugated AuNPs have been reported for the detection of polynucleotides and proteins (like p53, a tumor suppressor gene) with the help of various detection methods, for example, gel electrophoresis, chronocolormetry, atomic force microscopy, amplified voltametric detection, scanometric assay, SPR imaging and Raman spectroscopy (1). Gold nanoparticles having a glutathione cap (GSH) with $\mathrm{COOH}$ grouping and fluorescein isothiocynate (FITC) tag along with folic acid were used to target carcinoma cells. Due to the expression of folic acid receptors on the HeLa cells, the AuNPs interact only with them, thus, providing a sensitive and easy method for detection of cancer cells, and differentiating them from non-cancerous cells $(9,42,46-49)$.

The various neuroendocrine carcinomas overexpress somatostatin receptors. This trait has been exploited for the detection of these carcinomas by use of functionalized AuNPs with octreotide peptide, a synthetic analogue of somatostatin. The development of bioimaging agents based on the use of such receptors can help in tumor diagnosis at an early stage. Owing to the increased capacity of recognized as the protein receptor and enhanced fluorescence properties, octreotide functionalized AuNPs show more interaction than the AuNPs alone towards the tumor cells $(42-49,58)$. Likewise, coumarin dye and PEG AuNPs show effective internalization into the human breast cancer cells. The dual functionalization of fluorescent dye along with biomolecule can be used for bioimaging and subsequent drug delivery $(9,42-49)$.

Monoclonal antibodies ( $\mathrm{mAb}$ ) conjugation has also been helpful in the imaging of carcinomas. A PEG modified nanoparticle with covalently conjugated mAb Herceptin has enabled detection of breast carcinoma. Similarly, other mAb can also be used for diagnosis of various cancers $(3,9)$.

There are other techniques for visualization of carcinomas, including Multiphoton Plasmon resonance microscopy, photoacoustic tomography (PAT), third-harmonic microscopy and optical coherence microscopy. These are new and promising techniques in the field of cancer diagnosis (9). PAT is a hybrid imaging technique that utilises light for rapid heating of elements within the tissue, leading to the production of photoacoustic wave (because of thermoelastic expansion) generation that can be detected with an ultrasonic transducer. The NIR-absorbing gold nanoparticles can significantly enhance the image contrast when used in this technique, due to the more considerable differences in optical absorption (hence generating a stronger photoacoustic wave) than the endogenous tissue chromophores. Raman spectroscopy is another but most promising imaging technique with respect to gold nanoparticlebased contrast agent. The SERS nanoparticles consisting of a gold core, a Raman-active molecular layer, and an outermost silica coating were used for Raman imaging in vivo. Multiple SERS nanoparticles with different NIR absorption wavelengths can allow concurrent imaging of many tumor markers, and may have considerable potential in clinical applications (1).

\section{Cancer therapy}

Conventional treatment methods for cancer therapy include radiation therapy, chemotherapy and surgery. AuNPs due to their unique properties have received most attention for cancer therapy (1). The AuNP conjugates have been studied for enhancement in outcomes of radiotherapy as well as in a light-based therapy against cancer. Use of conjugated AuNPs ensures selective destruction of the targeted tumor cells. Radiotherapy has been widely used tool for cancer treatment in the past with the main objective for destruction of cancer cell DNA strands. The goal was achieved through irradiation of cancer cells by focussing highly energetic and penetrating gamma-rays, X-rays, photon and electron radiations. AuNPs have a higher probability of absorption of radiation and production of photoelectrons, X-ray fluorescence and Auger electrons as they contain heavy atoms with a large amount of electrons. Photoelectrons have better penetration through cells to produce their cytostatic effects. Thus, it provides an enhancement to the dose effectiveness of radiation in comparison to radiotherapy used solely (59). Unfortunately, the normal cells are also adversely affected by radiotherapy.

The local surface plasmon resonance property of AuNPs 
was explored for therapeutic purposes due to photothermal therapy (PTT) of tumor cells and design of light-triggered drug delivery (LTDD) system (59). In photothermal therapy, upon exposure to NIR light, the AuNPs convert absorbed light to heat via electron-electron collision leading to hot electrons. This heating effect with the use of AuNPs can cause cell death due to the alteration of normal cellular mechanism via apoptotic mechanism. A further increase in heating could result in cell cycle disruption $(10,11,16,59)$. There are in vitro studies that illustrate the use of various antibodies which are capable of producing selective destruction of the targeted cell. A comparison amongst the various types of nanoparticles, gold nanorods shows optimal light absorption and heat transduction. Also, they are the most efficient photothermal contrast agents, with the highest efficiency of light absorption and heat transduction.

Another application of optical properties of AuNPs is LTDD. In this system, AuNPs should be capped with agents such as biomolecules, polymers, molecular species, etc., which can act as reservoirs for the heat-generated release of chemotherapeutic drug on irradiation. These cytotoxic drugs can be loaded onto the nanoparticles by use of several strategies such as surface modification of the AuNPs to allow interaction with the drug that either supramolecular or electrostatic, hollow nanostructures designed to store drug in them like nanoshells and nanocages and insertion of nanoparticles into a highly thermosensitive structure like liposomes and temperature responsive hydrogels. The best designed photoactive Aubased nanocarriers is gold nanocage-thermosensitive gel and porous-gold-nanoshell-PEG. They are capable of storing large amounts of almost any drug molecule and are biologically inert. Both of these designs are NIR photoactive, with sizes $(40-60 \mathrm{~nm})$ that allow using the EPR effect to reach the tumor cells (59).

\section{Detection of biological molecules}

Based on the divergent and unique properties as well as using various strategies, AuNPs may be used for the detection of biological molecules like enzymes, DNA, proteins, antigens and antibodies $(13,29)$. The AuNP's use in detection of oligonucleotide was first found by Mirkin and colleagues. They found that single stranded oligonucleotide targets could be detected by use of two dissimilar Au-nanoprobes (thiol-linked functionalized oligonucleotide). Each of the Au-nanoprobes was functionalized with oligonucleotides that were complementary to one of the target oligonucleotide (38).
Upon introduction of the target into the Au-nanoprobe solution resulted in a colour change from red-to-blue. The change was due to formation of polymeric network (cross linking mechanism), which led to the nanoparticles close enough to produce colour change. This method has been expanded to a real time screening assay for endonuclease activity. Another easy-to-use and inexpensive assay was discovered which utilizes an increase in the salt concentration to induce aggregation to Au-nanoprobes. Here, the aggregation of Aunanoprobes does not result in the presence of complementary strands, rather occurs when non-complementary or mismatched targets are present. This method was also used in the detection of eukaryotic gene expression without the use of PCR amplification or retro-transcription steps $(18,38)$.

Another domain for the use of AuNPs is for detection of proteins on the basis of their characteristic surface plasmons. For this purpose a bifunctional molecule is needed which is conjugated to AuNP on one side via thiol conjugation and on the other side to electron rich aromatic side of protein via diazonium moiety. The nanoparticle acting as a Raman marker enhances the vibration of the diazo-bond between the protein and the bifunctional molecule. Finally, protein could be detected by using surface enhanced Raman spectroscopy. Thrombin was used as a protein to test this method (9, 42-49).

A recent involvement of the Au-nanoprobe cross-linking method is a strategy called bio-barcode assay. For the detection of proteins it shows attomolar sensitivity, while for DNA detection it has zeptomolar sensitivity (18). In this method, the analyte either protein or DNA is attached to a magnetic microparticle displaying recognition elements. Next, it attachs to a functionalised AuNP having a second recognition agent and 'barcode' DNA strands which are the markers, forming a sandwich complex (72). Separation of the complex is done by means of magnetic methods. In the end, the DNA barcodes are released and the analyte which is either a protein or a DNA is detected as well as quantified by the use of Au-nanoprobe sandwich assay and scanometric detection (using silver enhancement). The conventional bio-barcode assays are expensive and laborious, and depending upon sophisticated instruments, modifications have been developed that utilizes colorimetric, electrochemical, chemiluminescent and fluorimetric detection techniques (18). A successful use of this method has been studied in the detection and measurement of concentrations of amyloid- $\beta$-derived diffusible ligand, a potential marker present at cerebrospinal fluid (CSF) of Alzheimer's disease affected patients (72). 
Table 1. Biomedical applications of polymeric nanomaterial loaded with AuNPs

\begin{tabular}{|c|c|c|}
\hline $\begin{array}{c}\text { Polymeric nanomaterial loaded } \\
\text { with AuNPs }\end{array}$ & Applications & Reference \\
\hline $\begin{array}{c}\text { 5-Aminolevulinic acid } \\
\text { incorporated poly (vinyl alcohol) } \\
\text { nanofibre }\end{array}$ & Colon cancer & (60) \\
\hline Indomethacin-eluting stent & Tracheal regeneration & (61) \\
\hline $\begin{array}{l}\text { PLGA/collagen with PLGA/drug as } \\
\text { nano-fibre core }\end{array}$ & Repairing/healing wounds & (62) \\
\hline Drug-eluting nanofibres & Contraception & (63) \\
\hline $\begin{array}{l}\text { Biodegradable PLGA nano- } \\
\text { fibrous scaffolds with Vancomycin }\end{array}$ & Brain tissue drug delivery & $(64,65)$ \\
\hline $\begin{array}{l}\text { Biodegradable PLGA nano- } \\
\text { fibrous scaffolds with lidocaine }\end{array}$ & Epidural space drug delivery applications & (66) \\
\hline $\begin{array}{l}\text { Paclitaxel-eluting PCL } \\
\text { nano-fibrous structure }\end{array}$ & Benign cardiac stricture & (67) \\
\hline $\begin{array}{l}\text { AuNPs loaded BSA/PVA } \\
\text { nano-fibrous scaffolds }\end{array}$ & Cardiac tissue regeneration & (68) \\
\hline $\begin{array}{c}\text { AuNPs incorporated in } \\
\text { polymethylglutarimide (PMGI) } \\
\text { nano-fibers }\end{array}$ & $\begin{array}{c}\text { Enhancing attachment and } \\
\text { differentiation of mammalian cells, } \\
\text { enhancing HeLa cell attachment and } \\
\text { potentiating } \\
\text { cardiomyocyte differentiation of human } \\
\text { pluripotent stem cells }\end{array}$ & (69) \\
\hline $\begin{array}{l}\text { Chitin nano-fibers (CNFs) with } \\
\text { metallic gold nanoparticles } \\
\text { (AuNPs) }\end{array}$ & $\begin{array}{l}\text { Cosmetic, pharmaceuticals, catalysts, } \\
\text { electrical, electronic and optical devices. }\end{array}$ & (70) \\
\hline $\begin{array}{l}\text { Stretchable poly ( } \epsilon \text {-caprolactone) } \\
(\mathrm{PCL}) \text { gold nano particles coated } \\
\text { force sensors }\end{array}$ & Hand rehabilitation & $(70,71)$ \\
\hline
\end{tabular}

Another usefulness of AuNPs has been for the detection of biologically active materials such as vitamin $\mathrm{E}$ and aflatoxin A. Vitamin E analogue functionalised with AuNPs via selfassembly of thiol ligand was used to evaluate the free radical scavenging activity. For detection of aflatoxins, antibodies against AFB1 functionalised on AuNPs using electrodeposition on cysteamine functionalized AuNPs were used. Detection of aflatoxin AFB1 was highly efficient and required less response time $(9,46-49)$.

\section{Detection of microorganisms}

AuNPs have been used for the detection bacteria and viruses by using various biological, molecular and microbiological methods (18, 42-49). An application for the use of Au-nanoprobes was for the detection of Microbacterium tuberculosis (MTB) and its complex (MTBC). This consists of a AuNP-based colorimetric assay which involves detection of nested PCR-amplified DNA targets of MTB and MTBC by using two probes generated with thiol-modified oligonucleotides conjugated AuNPs. On hybridisation of the two probes with their target caused aggregation of AuNPs and consequently resulted in change of colour from red to blue/purple.

A similar colorimetric assay was developed for detection 
of unamplified HCV (define HCV) RNA from clinical specimens. After extraction of HCV RNA from the patient serum, it was mixed with a specific oligonucleotide sequence in a salt containing hybridization buffer. Further, the mixture was denatured, annealed, and cooled to room temperature, then unmodified $15 \mathrm{~nm}$ AuNPs were added to it. The solution changed its colour to blue if there was aggregation of AuNPs in $\mathrm{HCV}$ positive specimens, while remained red in the negative specimens. There are various other examples for detection of microorganisms with the use of AuNPs (18, 47-49).

\section{AuNP biosensors for detection of disease etiology}

An array of biosensors has been developed for basic research and application of AuNPs for detecting specific biomolecules involved in disease etiology. A nanosensor was used for the detection of cholesterol by immobilizing cholesterol oxidase on the basis of amperometric changes (9, 42-49, 73). The nanosensors used were made from gold-platinum alloy nanoparticles and showed to have high sensitivity, selectivity, fast response and good reproducibility. The principle of the detection was based upon hydrogen peroxide activity. Further, AuNP can aid detection of uric acid. Uric acid is a crucial metabolite of purines and its abnormal levels can lead to various metabolic disorders like cardiovascular diseases, gout, kidney damage, pneumonia, hyperuriceamia, and Lesch-Nyhan syndrome. The detection can be achieved by using amperometric methods, which are superior to several other methods, including enzymatic, colorimetric and electrochemical methods in a way that it can detect uric acid in whole blood, serum, and urine with detection limit as low as $50 \mathrm{nM}(9,74)$. Also, AuNPs have been developed for the determination of choline in various human samples as well as in colorimetric biosensors for the detection of proteinase activity assay $(9,43-48)$.

Several 'universal' colorimetric biosensor methods have been devised for the detection of a wide range of targets including proteins, nucleic acids, DNA, small molecules, and ions. Unmodified AuNPs, ssDNA probe, and a conjugated water soluble cationic polyelectrolyte are used in this biosensor. The colourimetric technique employed in the detection is the sequestration of the conjugated polyelectrolyte to ssDNA probe that causes aggregation of the AuNPs to produce a colour change from red to blue. This happens because the polyelectrolyte is not present to stabilise the nanoparticle. When a complementary target is present, a weak bond is formed between the probe target dsDNA and conjugated polyelectrolyte. Now, the polyelectrolyte remains free to stabilise the AuNP against aggregation. Target concentration can be determined by the use of absorbance ratio. This method has also been used to detect the aptamers which bind to specific molecular targets. Thrombin aptamer, anti-cocaine aptamer, and a mercury-responsive sequence that folds in the presence of $\mathrm{Hg}$ (II) were used in this assay for the detection of their respective targets (18).

\section{Single nucleotide polymorphisms detection}

Single nucleotide polymorphisms (SNPs) detection is also an extension of the AuNP's applications. This method is appropriate for the detection of point mutation and polymorphisms in various genes associated with diseases and metabolic disorders. The technique is used to assist in finding the explanation behind individual genetic variability, which is correlated with individual susceptibility to various diseases like $\beta$-thalessemia, diabetes mellitus, some cancers and individual responses to therapeutic agents. One of the most successful uses of nanoprobes method was in the clinical diagnosis of Mycobaterial tuberculosis. Another system utilised functionalised AuNP for the detection and characterisation of human $\mathrm{p} 53$ gene. This technique depicts potential for cancer diagnosis $(9,18,72)$.

\section{Immunoassay}

AuNPs have been applied in the designing of various immunoassays. They have shown potential in the enhancement of standard enzyme-linked immunosorbent assays (ELISA) signals via conjugation with antibodies or coupling with sliver enhancement. Besides ELISA, other techniques such as electrochemical methods, conductometery, colorimetry etc. have also been used for the detection purposes. AuNPs have been studied for the novel enhancement of immunochromatographic strips which increase the detection limit of hCG (72). Here, both the primary and the secondary antibodies were conjugated to AuNPs. A biochip detection of protein was designed using AuNPs and silver enhancement. In this procedure antigen is sandwiched in between immobilised antibody (antibody immobilised onto the surface of a chip with an electrode array using standard micro-electro mechanical systems-MEMS technology) and another AuNP labelled antibody. On addition of silver enhancement solution, reduction of silver ions to metallic silver with hydroquinone catalysed by AuNP occurs, followed by deposition of metallic silver onto the surface of AuNPs increasing the size of the particles. The final product results in the easy detection of 
change in electric conduction and determination of protein via conductometric methods (18).

The new sensing platforms were designed to replace the standard ELISA method as they do not demand secondary antibody as well as allows increase in sensitivity. The use of mono- and poly-clonal antibodies has been replaced by the immunosensors utilising single chain fragment variable recombinant antibodies $(\mathrm{scFv})$. These scFvs are small heterodimers consisting of antibodies with both variable heavy (VH) and light ( $\mathrm{LH}$ ) chains linked by a stabilising peptide linker. A colorimetric assay was developed with the use of $\mathrm{scFv}$ functionalised AuNPs having a cysteine or histidine in its linker region. The application of AuNPs in another immunoassay is under development and is considered to have a great potential for future use $(9,75)$.

\section{Enzyme immobilisation}

Enzyme immobilisation matrices have been made using AuNPs. The ability of the AuNPs is utilised to attach and functionalise the enzymes, and this process makes the enzymes more thermally stable in comparison to the free enzyme. Additionally, hollow nanospheres containing active enzymes have been synthesised. By this method, enzymes remain active for a long period of time and it helps in the detection of small molecules which can enter in the nanoshells $(9,43-50)$.

\section{CONCLUSION}

The novel development of AuNPs for drug or gene carriers and as molecular nanoprobes provides a useful bridging tool over the traditional delivery vehicles. AuNPs represent an immense scope in biomedical applications due their unique properties of high surface/volume ratio, low inherent toxicity and surface engineering. The availability of different synthetic methods for creating AuNPs of different sizes and shapes bestows a versatile toolbox for the fabrication of conjugates with enhanced affinity for targeted cell receptors, coherent internalisation, high biocompatibility, enhanced permeation into tumor cells, and long circulation half-life $\left(t_{1 / 2}\right)$ of conjugates or medicaments. Furthermore, the optical and electronic properties of AuNPs are extremely useful for monitoring and detecting the biological molecules microbes of interest. Recent research on the successful utilisation of AuNPs in diagnosis of some diseases and targeted drug delivery system has set a platform for the future development of new applications in biomedical sciences. Overall, the AuNPs offer a bright scope for the diagnosis and treatment of diseases.
However, there are important issues that need attention such as immune response, unwanted cytotoxicity, and long-term adverse consequences.

Conflict of interest: the authors declare no conflict of interest.

\section{REFERENCES}

1. Cai W, Gao T, Hong H, Sun J. Application of Au nanoparticles in cancer nanotechnology. Nanotechnol Sci Appl 2008; 1:17-32. DOI: 10.2147/NSA.S3788

2. Pankhurst QA, Connolly J, Jones SK, Dobson J. Applications of magnetic nanoparticles in biomedicine. J Phys Appl Phys 2003; 36:R167-R181. DOI: 10.1088/0022-3727/36/13/201

3. Mazzola L. Commercializing nanotechnology. Nat Biotechnol 2003; 21:1137-1143. DOI:10.1038/NBT10031137

4. Parak WJ, Gerion D, Pellegrino T, Zanchet D, Micheel C, Williams CS, et al. Biological applications of colloidal nanocrystals. Nanotechnology 2003; 14:R15-R27. DOI:10.1088/0957-4484/14/7/201

5. Nam JM, Thaxton CC, Mirkin CA. Nanoparticlesbased bio-bar codes for the ultrasensitive detection of proteins. Science 2003; 301:1884-1886. DOI: 10.1126/ science. 1088755

6. Reich DH, Tanase M, Hultgren A, Bauer LA, Chen CS, Meyer GJ. Biological applications of multifunctional magnetic nanowires. J Appl Phys 2003; 93:7275-7280. DOI: $10.1063 / 1.155867$

7. El Fray M, Strzalkowska D, Mandoli C, Pagliari F, Di Nardo P, Traversa E. Influence of ceria nanoparticles on chemical structure and properties of segmented polyesters. Mater Sci Eng C Mater Biol Appl 2015; 53:15-22. DOI: 10.1016/j.msec.2015.04.010

8. Roy I, Ohulchanskyy TY, Pudavar HE, Bergey EJ, Oseroff AR, Morgan J, et al. Ceramic-based nanoparticles entrapping water-insoluble photosensitizing anticancer drugs: a novel drug-carrier system for photodynamic therapy. J Am Chem Soc 2003; 125:7860-7865. DOI: 10.1021/ja0343095

9. Raghavendra R, Arunachalam KD, Annamalai SK, Arunachalam AM. Diagnostics and therapeutic application of gold nanoparticles. Int J Pharm Pharm Sci 2014; 6:74-87.

10. Huang X, El-Sayed MA. Gold nanoparticles: Optical properties and implementations in cancer diagnosis and photothermal therapy. $J$ Adv Res 2010; 1:13-28. DOI:10.1016/j.jare.2010.02.002 
11. Huang X, Jain PK, El-Sayed IH, El-Sayed MA. Gold nanoparticles: interesting optical properties and recent applications in cancer diagnostics and therapy. Nanomedicine 2007; 2:681-693. DOI:10.2217/17435889.2.5.681

12. Giljohann DA, Seferos DS, Daniel WL, Massich MD, Patel PC, Mirkin CA. Gold nanoparticles for biology and medicine. Angew Chem Int Ed 2010; 49:3280-3294. DOI: 10.1002/anie.200904359

13. Parida UK, Nayak PL. Biomedical applications of gold nanoparticles: Opportunity and challenges. World $J$ Nano Sci Technol 2012; 1:10-25. DOI: 10.5829/idosi. wjnst.2012.1.2.202

14. Das M, Shim KH, An SSA, Yi DK. Review on gold nanoparticles and their applications. Toxicol Envir Health Sci 2011; 3:193-205. DOI: 10.1007/s13530-011-0109-y

15. Faraday M. The Bakerian Lecture: Experimental Relations of Au (and Other Metals) to Light. Phil Trans R Soc Lond 1857; 147:145-181.

16. Voliani V, Signore G, Nifosí R, Ricci F, Luin S, Beltram F. Smart Delivery and Controlled Drug Release with Gold Nanoparticles. New Front Nanomed Recent Patents Nanomed 2012; 2:34-44. DOI: 10.2174/1877912311202010034

17. Zhou J, Ralston J, Sedev R, Beattie DA. Functionalized gold nanoparticles: Synthesis, structure and colloid stability. J Colloid Interface Sci 2009; 331:251-262. DOI: 10.1016/j.jcis.2008.12.002.

18. Azzazy HME, Mansour MMH, Samir TM, Ricardo F. Gold nanoparticles in the clinical laboratory: principles of preparation and applications. Clin Chem Lab Med 2012; 50:193-209. DOI: 10.1515/CCLM.2011.732.

19. de la Fuente JM, Grazu V. Inorganic nanoparticles vs organic nanoparticles. Nanobiotechnology 2012; 4:3-30.

20. Jana NR, Gearheart L, Murphy CJ. Seed-mediated growth approach for shape-controlled synthesis of spheroidal and rod-like gold nanoparticles using a surfactant template. Adv Mater 2001;13:1389-1393. DOI: 10.1002/15214095(200109)13:18<1389

21. Busbee BD, Obare SO, Murphy CJ. An improved synthesis of high aspect-ratio gold nanorods. Adv Mater 2003;15:414-416. DOI: 10.1002/adma.200390095

22. Jana NR, Gearheart L, Murphy CJ. Wet chemical synthesis of high aspect ratio cylindrical gold nanorods. J Phys Chem B 2001; 105:4065-4067. DOI: $10.1021 /$ jp0107964
23. Brinson BE, Lassiter JB, Levin CS, Bardhan R, Mirin N, Halas, Naomi J. Nanoshells Made Easy: Improving Au Layer Growth on Nanoparticle Surfaces. Langmuir 2008; 24:14166-14171. DOI: 10.1021/la802049p

24. Chen J. et al. Facile synthesis of Au-silver nanocages with controllable pores on the surface. J Am Chem Soc 2006; 128:14776-14777. DOI: $10.1021 / \mathrm{ja} 066023 \mathrm{~g}$

25. Kim CK, Ghosh P, Rotello VM. Multimodal drug delivery using gold nanoparticles. Nanoscale 2009; 1:61-67. DOI: $10.1039 / \mathrm{B} 9 \mathrm{NR} 00112 \mathrm{C}$

26. Kam NWS, Jessop TC, Wender PA, Dai H. Nanotube molecular transporters: internalization of carbon nanotube protein conjugates into mammalian cells. J Am Chem Soc 2004; 126:6850-6851. DOI: 10.1021/ja0486059

27. Chithrani BD, Ghazani AA, Chan WCW. Determining the size and shape dependence of gold nanoparticle uptake into mammalian cells. Nano Lett 2006;6:662-668. DOI: $10.1021 / \mathrm{n} 10523960$

28. Mukherjee S, Ghosh RN, Maxfield FR. Endocytosis. Physiol Rev 1997;77:759-803.

29. Kirchhausen T. Three ways to make a vesicle. Nat Rev Mol Cell Biol. 2000; 1:187-198. DOI:10.1038/35043117

30. Chithrani DB. Intracellular uptake, transport, and processing of gold nanostructures. Mol Memb Biol 2010; 27:299-311. DOI: 10.3109/09687688.2010.507787

31. Arvizo R, Bhattacharya R, Mukherjee P. Gold nanoparticles: opportunities and challenges in nanomedicine. Expert Opin Drug Deliv 2010;7:753-763. DOI: $10.1517 / 17425241003777010$

32. Gibson JD, Khanal BP, Zubarev ER. Paclitaxelfunctionalized gold nanoparticles. J Am Chem Soc 2007; 129:11653-11661. DOI: 10.1021/ja075181k

33. Anderson ME. Glutathione: an overview of biosynthesis and modulation. Chem. Biol. Interact. 1998;112:1-14. DOI:10.1016/S0009-2797(97)00146-4

34. Sies H. Glutathione and its role in cellular functions. Free Radic Biol Med 1999;27:916-921. DOI:10.1016/S08915849(99)00177-X

35. McIntosh CM, Esposito EA, Boal AK, Simard JM, Martin CT, Rotello VM. Inhibition of DNA transcription using cationic mixed monolayer protected gold clusters. $J \mathrm{Am}$ Chem Soc 2001;123:7626-7629. DOI: 10.1021/ja015556g

36. Verma A, Simard JM, Worrall JWE, Rotello VM. Tunable reactivation of nanoparticle-inhibited beta-galactosidase by glutathione at intracellular concentrations. $\mathrm{J} \mathrm{Am} \mathrm{Chem}$ Soc 2004;126:13987-13991. DOI: 10.1021/ja046572r 
37. Bhumkar DR, Joshi HM, Sastry M, Pokharkar VB. Chitosan reduced gold nanoparticles as novel carriers for transmucosal delivery of insulin. Pharm Res 2007;24:1415-1426. DOI: 10.1007/s11095-007-9257-9

38. Jeong EH, Jung G, Hong C Am, Lee H. Gold nanoparticle (AuNP)-based drug delivery and molecular imaging for biomedical applications. Arch Pharm Res 2014;37:53-59. DOI: $10.1007 / \mathrm{s} 12272-013-0273-5$

39. Ghosh P, Han G, De M, Kim CK, Rotello VM. Gold nanoparticles in delivery applications. Adv Drug Deliv Rev 2008; 60:1307-1315. DOI:10.1016/j.addr.2008.03.016

40. Han G, Ghosh P, Rotello VM. Functionalized gold nanoparticles for drug delivery. Nanomedicine 2007; 2:113-123.

41. Kim CK, Ghosh P, Rotello VM. Multimodal drug delivery using gold nanoparticles. Nanoscale 2009; 1:61-67. DOI: 10.1039/B9NR00112C

42. Lee SH, Bae KH, Kim SH, Lee KR, Park TG. Amine functionalized gold nanoparticles as non-cytotoxic and efficient intracellular siRNA delivery carriers. Int J Pharm 2008; 364:94-101. DOI:10.1016/j. ijpharm.2008.07.027

43. Wangoo N, Bhasin KK, Mehta SK, Suri CR. Synthesis and capping of water-dispersed gold nanoparticles by an amino acid: Bioconjugation and binding studies. $J$ Colloid Interface Sci 2008; 323:247-254. DOI:10.1016/j. jcis.2008.04.043

44. Lee JS, Green JJ, Love KT, Sunshine J, Langer R, Anderson DG. Gold, poly( $\beta$-amino ester) nanoparticles for small interfering RNA delivery. Nano Lett 2009; 9:24022406. DOI: 10.1021/n19009793.

45. Giljohann DA, Seferos DS, Prigodich AE, Patel PC, Mirkin CA. Gene regulation with polyvalent siRNAnanoparticle conjugates. J Am Chem Soc 2009;131:20722073. DOI: $10.1021 /$ ja808719p

46. Guo S, et al. Enhanced gene delivery and siRNA silencing by gold nanoparticles coated with charge-reversal polyelectrolyte. ACS NANO 2010; 4:5505-5511. DOI: $10.1021 / \mathrm{nn} 101638 \mathrm{u}$.

47. Davis ME et al. Evidence of RNAi in humans from systemically administered siRNA via targeted nanoparticles. Nature 2010;464:1067-1070. DOI:10.1038/ nature 08956

48. Sharma A, Tandon A, Tovey JC, Gupta R, Robertson JD, Fortune JA, Klibanov AM, Cowden JW, Rieger FG, Mohan RR. Polyethylenimine-conjugated gold nanoparticles: Gene transfer potential and low toxicity in the cornea. Nanomedicine 2011; 7:505-513. DOI:10.1016/j. nano.2011.01.006.

49. Fortune JA, Novobrantseva TI, Klibanov AM. Highly effective gene transfection in vivo by alkylated polyethylenimine. J Drug Deliv 2011;204058. DOI:10.1155/2011/204058

50. Suzawa T, Nagamura S, Saito H, Ohta S, Hanai N, Kanazawa J, et al. Enhanced tumor cell selectivity of adriamycin-monoclonal antibody conjugate via a poly(ethylene- glycol)-based cleavable linker. $J$ Contr Rel 2002;79:229-242. DOI:10.1016/S01683659(01)00554-5

51. Kohler G, Milstein C. Continuous cultures of fuse cells secreting antibody of predefined specificity. Nature 1975; 256:495-497. DOI:10.1038/256495a0

52. Abou-Jawde R, Choueiri T, Alemany C, Mekhail T. An overview of targeted treatments in cancer. Clin Ther 2003;25:2121-2137. DOI:10.1016/S01492918(03)80209-6

53. Glennie MJ, van de Winkel JGJ. Renaissance of cancer therapeutic antibodies. Drug Discov Today 2003;8:503510. DOI:10.1016/S1359-6446(03)02714-4

54. Wang YM, Sato H, Dachi I, Horikoshi I. Preparation and characterization of poly(lactic-co-glycolic acid) microspheres for targeted delivery of novel anticancer agent, taxol. Chem Pharm Bull 1996; 44:1935-1940. DOI: $10.1248 / \mathrm{cpb} .44 .1935$

55. Si-Shen F, Guofeng H. Effect of emulsifiers on the controlled release of paclitaxel (Taxol) from nanospheres of biodegradable polymer. J Contr Rel 2001; 71:53-69. DOI:10.1016/S0168-3659(00)00364-3

56. Williams J, Lansdown R, Sweitzer R, Romanowski M, LaBell R, Ramaswami R, et al. Nanoparticle drug delivery system for intravenous delivery of topoisomerase inhibitors. J Contr Rel 2003;91:167-172. DOI:10.1016/ S0168-3659(03)00241-4

57. Dagar S, Krishnadas A, Rubinstein I, Blend MJ, Onyüksel H. VIP grafted sterically stabilized liposomes for targeted imaging of breast cancer: in vivo studies. J Contr Release 2003;91:123-133. DOI:10.1016/S0168-3659(03)00242-6

58. Surujpaul PP, Gutiérrez-Wing C, Ocampo-García B, de M. Ramírez F, Arteaga de Murphy C, Pedraza-López M, et al. Gold nanoparticles conjugated to [Tyr3] Octreotide peptide. Biophys Chem 2008;138(3):83-90. DOI: 10.1016/j.bpc.2008.09.005 
59. Cao-Milán R, Liz-Marzán, LM. Gold nanoparticle conjugates: recent advances toward clinical applications. Expert Opin Drug Deliv 2014;11:741-752. DOI: $10.1517 / 17425247.2014 .891582$

60. Lia Y, Schluesenerb HJ, Xu S. Gold nanoparticle-based biosensors. Gold Bull 2010; 43:29-41. DOI: 10.1007/ BF03214964

61. Yoo JJ, Kim C, Chung CW, Jeong YI, Kang DH. 5-aminolevulinic acid-incorporated poly (vinyl alcohol) nanofiber-coated metal stent for application in photodynamic therapy. Int J Nanomed 2012; 7:1997-2005. DOI: $10.2147 /$ IJN.S30298

62. Heo DN, Lee JB, Bae MS, Hwang YS, Kwon KH, Kwon IK. Development of nanofiber coated indomethacin-eluting stent for tracheal regeneration. J Nanosci Nanotechnol 2011;11:5711-5716. DOI: 10.1166/jnn.2011.4495

63. Chen DW, Liao JY, Liu SJ, Chan EC. Novel biodegradable sandwich-structured nanofibrous drug-eluting membranes for repair of infected wounds: an in vitro and in vivo study. Int J Nanomed 2012; 7:763-771. DOI: 10.2147/IJN.S29119

64. Ball C, Krogstad E, Chaowanachan T, Woodrow

KA. Drug-eluting fibers for HIV-1 inhibition and contraception. PLoS One 2012;7:e49792. DOI:10.1371/ journal.pone.0049792

65. Tseng YY, Kao YC, Liao JY, Chen WA, Liu SJ. Biodegradable drug-eluting poly[lactic-coglycol acid] nanofibers for the sustainable delivery of vancomycin to brain tissue: in vitro and in vivo studies. ACS Chem Neurosci 2013; 4:1314-1321. DOI: 10.1021/cn400108q

66. Tseng YY, Liao JY, Chen WA, Kao YC, Liu SJ. Biodegradable poly([D,L]-lactide-co-glycolide) nanofibers for the sustainable delivery of lidocaine into the epidural space after laminectomy. Nanomedicine 2014; 9:77-87. DOI: $10.2217 / \mathrm{nnm} .13 .42$

67. Zhu YQ, Cui WG, Cheng YS, Chang J, Chen NW, Yan L. Evaluation of biodegradable paclitaxel eluting nanofibrecovered metal stents for the treatment of benign cardiac stricture in an experimental model. Br J Surg 2013;
100:784-793. DOI: 10.1002/bjs.9106.

68. Ravichandran R, Sridhar R, Venugopal JR, Sundarrajan S, Mukherjee S, Ramakrishna S. Gold nanoparticle loaded hybrid nanofibers for cardiogenic differentiation of stem cells for infarcted myocardium regeneration. Macromol Biosci 2014;14:515-525. DOI: 10.1002/ mabi.201300407

69. Jung D, Minami I, Patel S, et al. Incorporation of functionalized gold nanoparticles into nanofibers for enhanced attachment and differentiation of mammalian cells. J Nanobiotechnol 2012; 10:23. DOI:10.1186/14773155-10-23

70. Shervani Z, Taisuke Y, Ifuku S, Saimoto H, Morimoto M. Preparation of gold nanoparticles loaded chitin nanofiber composite. Adv Nanopart 2012; 1:71-78. DOI: 10.4236/ anp.2012.13010

71. Ko J, Bhullar S, Cho Y, Lee PC, Jun MBG. Design and fabrication of auxetic stretchable force sensor for hand rehabilitation. Smart Mater Struct 2015; 24: 075027. DOI:10.1088/0964-1726/24/7/075027

72. Baptista P, Pereira E, Eaton P, Doria G, Miranda A, Gomes I, et al. Gold nanoparticles for the development of clinical diagnosis methods. Anal Bioanal Chem 2008; 391:943950. DOI: 10.1007/s00216-007-1768-Z

73. Safavi A, Farjami F. Electrodeposition of gold-platinum alloy nanoparticles on ionic liquid-chitosan composite film and its application in fabricating an amperometric cholesterol biosensor. Biosens Bioelectr 2011;26:25472552. DOI: 10.1016/j.bios.2010.11.002

74. Kannan P, John SA. Determination of nanomolar uric and ascorbic acids using enlarged gold nanoparticles modified electrode. Anal Biochem 2009; 386:65-72. DOI: 10.1016/j.ab.2008.11.043

75. Liu Y, Liu Y, Mernaugh RL, Zeng X. Single chain fragment variable recombinant antibody functionalized gold nanoparticles for a highly sensitive colorimetric immunoassay. Biosens Bioelectr 2009; 24:2853-2857. DOI: 10.1016/j.bios.2009.02.016 